\title{
EXISTENCE OF EXTREMAL BELTRAMI COEFFICIENTS WITH NONCONSTANT MODULUS
}

\author{
GUOWU YAO
}

\begin{abstract}
Suppose that $[\mu]_{T(\Delta)}$ is a point of the universal Teichmüller space $T(\Delta)$. In 1998, Božin, Lakic, Marković, and Mateljević showed that there exists $\mu$ such that $\mu$ is uniquely extremal in $[\mu]_{T(\Delta)}$ and has a nonconstant modulus. It is a natural problem whether there is always an extremal Beltrami coefficient of constant modulus in $[\mu]_{T(\Delta)}$ if $[\mu]_{T(\Delta)}$ admits infinitely many extremal Beltrami coefficients; the purpose of this paper is to show that the answer is negative. An infinitesimal version is also obtained. Extremal sets of extremal Beltrami coefficients are considered, and an open problem is proposed. The key tool of our argument is Reich's construction theorem.
\end{abstract}

\section{$\S 1$. Introduction}

Suppose that $\mathfrak{D}$ is a Jordan domain in the complex plane $\mathbb{C}$, and let $w=f(z)$ be a quasiconformal mapping on $\mathfrak{D}$. The complex dilatation of $f$ is defined by

$$
\mu(z)=\frac{f_{\bar{z}}(z)}{f_{z}(z)}
$$

which is also called the Beltrami coefficient of $f$.

Let $M(\mathfrak{D})$ be the open unit ball of $L^{\infty}(\mathfrak{D})$. Let $z_{1}, z_{2}, z_{3}$ be three boundary points on $\partial \mathfrak{D}$. For a given $\mu \in M(\mathfrak{D})$, denote by $f^{\mu}$ the uniquely determined quasiconformal mapping of $\mathfrak{D}$ onto itself with complex dilatation $\mu$ and normalized to fix $z_{1}, z_{2}, z_{3}$. The elements of $M(\mathfrak{D})$ are also called Beltrami coefficients. Two elements $\mu$ and $\nu$ in $M(\mathfrak{D})$ are Teichmüller-equivalent, which is denoted by $\mu \sim \nu$, if $\left.f^{\mu}\right|_{\partial \mathfrak{D}}=\left.f^{\nu}\right|_{\partial \mathfrak{D}}$. Then $T(\mathfrak{D})=M(\mathfrak{D}) / \sim$ is the Teichmüller space of $\mathfrak{D}$. The equivalence class of the Beltrami coefficient zero is the base point of $T(\mathfrak{D})$.

Received February 15, 2008. Revised March 3, 2009. Accepted May 10, 2009.

2000 Mathematics Subject Classification. Primary 30C75; Secondary 30C62.

The author's research was supported by the Foundation for the Author of National Excellent Doctoral Dissertation grant no. 200518, and by the National Natural Science Foundation of China. 
Given that $\mu \in M(\mathfrak{D})$, we denote by $[\mu]_{T(\mathfrak{D})}$ the set of all elements $\nu \in$ $M(\mathfrak{D})$ equivalent to $\mu$, and we set

$$
k(\mu)=\inf \left\{\|\nu\|_{\infty}: \nu \in[\mu]_{T(\mathfrak{D})}\right\} .
$$

We say that $\mu$ is extremal (in $[\mu]_{T(\mathfrak{D})}$ ) if $\|\mu\|_{\infty}=k(\mu)$ and uniquely extremal if $\|\nu\|_{\infty}>k(\mu)$ for any other $\nu \in[\mu]_{T(\mathfrak{D})}$. Accordingly, $f^{\mu}$ is called an extremal (uniquely extremal) quasiconformal mapping for its boundary correspondence. Let $[\mu]_{T(\mathfrak{D})}^{*}$ denote the set of all extremal Beltrami coefficients in $[\mu]_{T(\mathfrak{D})}$.

Throughout this paper, let $A(\mathfrak{D})$ denote the Banach space of all holomorphic functions $\varphi$ in the domain $\mathfrak{D}$ with $L^{1}$-norm

$$
\|\varphi\|=\iint_{\mathfrak{D}}|\varphi(z)| d x d y<\infty .
$$

Two elements $\mu$ and $\nu$ in $L^{\infty}(\mathfrak{D})$ are infinitesimally equivalent, which is denoted by $\mu \approx \nu$, if $\iint_{\mathfrak{D}} \mu \phi d x d y=\iint_{\mathfrak{D}} \nu \phi d x d y$ for all $\phi \in A(\mathfrak{D})$. Denote by $N(\mathfrak{D})$ the set of all the elements in $L^{\infty}(\mathfrak{D})$ which are infinitesimally equivalent to zero. Then $B(\mathfrak{D})=L^{\infty}(\mathfrak{D}) / N(\mathfrak{D})$ is the tangent space of the Teichmüller space $T(\mathfrak{D})$ at the base point.

Given that $\mu \in L^{\infty}(\mathfrak{D})$, we denote by $[\mu]_{B(\mathfrak{D})}$ the set of all elements $\nu \in$ $L^{\infty}(\mathfrak{D})$ infinitesimally equivalent to $\mu$, and we set

$$
\|\mu\|_{B(\mathfrak{D})}=\inf \left\{\|\nu\|_{\infty}: \nu \in[\mu]_{B(\mathfrak{D})}\right\} .
$$

We say that $\mu$ is extremal (in $[\mu]_{B(\mathfrak{D})}$ ) if $\|\mu\|_{\infty}=[\mu]_{B(\mathfrak{D})}$ and uniquely extremal if $\|\nu\|_{\infty}>\|\mu\|_{\infty}$ for any other $\nu \in[\mu]_{B(\mathfrak{D})}$. Note that $\mu$ is also called an extremal Beltrami coefficient if it is extremal and $\|\mu\|_{\infty}<1$. Similarly, let $[\mu]_{B(\mathfrak{D})}^{*}$ denote the set of all extremal elements in $[\mu]_{B(\mathfrak{D})}$.

A Beltrami coefficient $\mu$ is said to be of constant modulus if it has the form

$$
\mu(z)=k \frac{\overline{\varphi(z)}}{|\varphi(z)|},
$$

where $k \in[0,1)$ is a constant and $\varphi$ is a complex-valued function in $\mathfrak{D}$ with $\varphi \neq 0$ almost everywhere. Particularly, if $\varphi \not \equiv 0$ is meromorphic in $\mathfrak{D}$, then $\mu$ is called a Teichmüller Beltrami coefficient.

Let $\Delta$ be the unit disk $\{|z|<1\}$. In this paper, unless otherwise specified, we restrict the considerations to the special case $\mathfrak{D}=\Delta$ in order to simplify exposition. 
For a given point $[\mu]_{T(\Delta)}$ in the universal Teichmüller space $T(\Delta)$, there are two cases for the extremal Beltrami coefficients among $[\mu]_{T(\Delta)}$. One is that there is a unique extremal Beltrami coefficient in $[\mu]_{T(\Delta)}$ which may be of constant modulus or not (see [1]). The other is that there is more than one extremal Beltrami coefficient in $[\mu]_{T(\Delta)}$. In the latter case, in fact there are infinitely many extremal Beltrami coefficients in $[\mu]_{T(\Delta)}$ (see [9], [2]). Moreover, in this setting there definitely exists an extremal Beltrami coefficient of nonconstant modulus in $[\mu]_{T(\Delta)}$ (see [7], [11], [12]).

Is there always an extremal Beltrami coefficient of constant modulus in $[\mu]_{T(\Delta)}$ if it contains infinitely many extremal Beltrami coefficients? This is a natural problem (also posed in [12]). The author in [10] recently constructed certain $[\mu]_{T(\Delta)}$ admitting infinitely many extremal Beltrami coefficients such that it contains no extremal Teichmüller Beltrami coefficients. Perhaps one still expects that $[\mu]_{T(\Delta)}$ contains at least an extremal Beltrami coefficient of constant modulus. However, the following counterexample theorem gives the converse answer.

TheOREM 1. There exists a point $[\mu]_{T(\Delta)}$ in the universal Teichmüller space $T(\Delta)$ admitting more than one extremal Beltrami coefficient, such that $[\mu]_{T(\Delta)}$ contains no extremal Beltrami coefficients of constant modulus.

COROLlary 1. There exists some $[\mu]_{T(\Delta)}$ in $T(\Delta)$ admitting more than one extremal Beltrami coefficient, such that $[\mu]_{T(\Delta)}$ contains no extremal Teichmüller Beltrami coefficients.

We also obtain an infinitesimal version of Theorem 1.

THEOREM 2. There exists a point $[\mu]_{T(\Delta)}$ in $B(\Delta)$ admitting more than one extremal Beltrami coefficient, such that $[\mu]_{T(\Delta)}$ contains no extremal Beltrami coefficients of constant modulus.

Corollary 2. There exists some $[\mu]_{B(\Delta)}$ in $B(\Delta)$ admitting more than one extremal Beltrami coefficient, such that $[\mu]_{B(\Delta)}$ contains no extremal Teichmüller Beltrami coefficients.

Delta inequalities are introduced in Section 2. Some preparations are done in Section 3. After giving Reich's construction theorem and its applications in Section 4, we present the proofs of our main results in Section 5. At the end, we consider the extremal sets of extremal Beltrami coefficients and pose an open problem.

The results as well as the method used here can be extended to more general hyperbolic Riemann surfaces and their Teichmüller spaces. 


\section{$\S 2$. Delta inequalities}

For $\mu \in L^{\infty}(\Delta), \phi \in A(\Delta)$, let

$$
\Lambda_{\mu}[\phi]=\iint_{\Delta} \mu(z) \phi(z) d x d y, \quad \text { and } \quad \lambda_{\mu}[\phi]=\operatorname{Re} \Lambda_{\mu}[\phi] .
$$

The functional $\delta=\delta_{\mu}$ is defined on $\Delta$ by

$$
\delta(\varphi)=\|\mu\|_{\infty}\|\varphi\|-\lambda_{\mu}[\varphi], \quad \varphi \in A(\Delta) .
$$

We say that $\mu \in L^{\infty}(\Delta)$ satisfies Reich's condition on a set $E \subset \Delta$ if there exists a sequence $\varphi_{n}$ in $A(\Delta)$ so that $\delta\left(\varphi_{n}\right) \rightarrow 0$ and $\liminf \left|\varphi_{n}(z)\right|>0$ for almost all $z$ in $E$. Meanwhile, $\varphi_{n}(z)$ is called a Reich's condition sequence for $\mu$ on $E$.

REMARK 1. A Reich's condition sequence is also called a delta sequence, which was first introduced in [5].

As is well known, a necessary and sufficient condition (a HamiltonKrushkal-Reich-Strebel condition) that a quasiconformal mapping $f$ is extremal (for its boundary values) is that its Beltrami coefficient $\mu$ has a so-called Hamilton sequence (see [8]), namely, a sequence $\left\{\phi_{n} \in A(\Delta):\left\|\phi_{n}\right\|=1, n \in\right.$ $\mathbb{N}$ \} such that

$$
\lim _{n \rightarrow \infty} \Lambda_{\mu}\left[\phi_{n}\right]=\lim _{n \rightarrow \infty} \iint_{\Delta} \mu \phi_{n}(z) d x d y=\|\mu\|_{\infty} .
$$

Now, we introduce Reich's delta inequality and the infinitesimal delta inequality on the unit disk $\Delta$. Their generalized forms play important roles in the joint work of [1].

Suppose that $\mu$ and $\nu$ are two equivalent Beltrami coefficients in the universal Teichmüller space $T(\Delta)$. Let $\widetilde{\mu}$ and $\widetilde{\nu}$ be the Beltrami coefficients of the quasiconformal mappings $f^{-1}$ and $g^{-1}$, respectively, where $f=f^{\mu}$ and $g=f^{\nu}$.

Delta inequality. If $\mu$ and $\nu$ are equivalent Beltrami coefficients in $T(\Delta)$ with

$$
\|\nu\|_{\infty} \leq k=\|\mu\|_{\infty}<1
$$

then

$$
\iint_{\Delta}\left|\frac{\widetilde{\mu}(f)-\widetilde{\nu}(f)}{1-\widetilde{\widetilde{\mu}(f)} \widetilde{\nu}(f)}\right|^{2}|\varphi| \leq C\left(k\|\varphi\|-\operatorname{Re} \iint_{\Delta} \mu \varphi\right),
$$

for all $\varphi$ in $A(\Delta)$. The constant $C$ depends only on $k=\|\mu\|_{\infty}$. 
INFINITESIMAL DELTA INEQUALITY. There exists a universal constant $C$ such that, for every pair of infinitesimally equivalent Beltrami coefficients $\mu$ and $\nu$ with

$$
\|\nu\|_{\infty} \leq\|\mu\|_{\infty}<\infty
$$

we have

$$
\iint_{\Delta}|\mu-\nu|^{2}|\varphi| \leq C\|\mu\|_{\infty}\left(\|\mu\|_{\infty}\|\varphi\|-\operatorname{Re} \iint_{\Delta} \mu \varphi\right)
$$

for all $\varphi$ in $A(\Delta)$. The constant $C$ is independent of $\mu$ and $\nu$.

\section{$\S 3$. Some preparations}

Let $\mathfrak{J}_{i} \varsubsetneqq \Delta(i=1,2, \ldots, m)$ be $m(m \in \mathbb{N})$ Jordan domains such that $\overline{\mathfrak{J}_{i}}$ $(i=1,2, \ldots, m)$ are mutually disjoint and such that $\Delta \backslash \bigcup_{1}^{m} \overline{\mathfrak{J}_{i}}$ is connected. Let $\mu$ be a Beltrami coefficient in $M(\Delta)$. Let $T\left(\mathfrak{J}_{i}\right)$ be the Teichmüller space of $\mathfrak{J}_{i}$, respectively.

Lemma 1. Let $\mathfrak{J}_{i} \varsubsetneqq \Delta(i=1,2, \ldots, m)$ be given as above, and let $\mathfrak{J}=$ $\bigcup_{1}^{m} \mathfrak{J}_{i}$. Let $\mu$ and $\nu$ be two equivalent Beltrami coefficients in $T(\Delta)$. In addition, suppose that $\mu(z)=\nu(z)$ for almost every $z \in \Delta \backslash \overline{\mathfrak{J}}$. Then, $f^{\mu}(z)=$ $f^{\nu}(z)$ for all $z$ in $\Delta \backslash \mathfrak{J}$, and hence $f^{\mu}(\overline{\mathfrak{J}})=f^{\nu}(\overline{\mathfrak{J}})$.

Proof. For the sake of convenience, let $f=f^{\mu}$ and let $g=f^{\nu}$. Let $\mu_{g \circ f^{-1}}(w)$ denote the Beltrami coefficient of $g \circ f^{-1}$. By a simple computation, we have

$$
\mu_{g \circ f^{-1}} \circ f(z)=\frac{1}{\tau} \frac{\mu(z)-\nu(z)}{1-\overline{\mu(z)} \nu(z)},
$$

where $\tau=\overline{f_{z}} / f_{z}$.

Thus, $\mu_{g \circ f^{-1}}(w)=0$ for almost all $w \in f(\Delta \backslash \overline{\mathfrak{J}})$, and hence $\Psi=g \circ f^{-1}$ is conformal on $\Delta \backslash \overline{\mathfrak{J}}$. Since $\left.\Psi\right|_{S^{1}}=\left.g \circ f^{-1}\right|_{S^{1}}=\mathrm{id}$, we conclude that $\Psi=\mathrm{id}$ in $f(\Delta \backslash \mathfrak{J})$. Furthermore, we conclude that $\left.\Psi\right|_{f(\partial \mathfrak{J})}=$ id by the continuity of quasiconformal mappings. Thus, $\left.g\right|_{\Delta \backslash \mathfrak{J}}=\left.f\right|_{\Delta \backslash \mathfrak{J}}$, which evidently gives the lemma.

From Lemma 1, we easily obtain the following. 
Lemma 2. Let $\mathfrak{J}_{i}(i=1,2, \ldots, m)$, and let $\mathfrak{J}$ be given as above. Suppose that $\mu(z)$ is a Beltrami coefficient in $M(\Delta)$. Let $\nu(z)$ be another Beltrami coefficient in $M(\Delta)$ defined as follows:

$$
\nu(z)= \begin{cases}\mu(z), & z \in \Delta \backslash \mathfrak{J}, \\ \beta_{i}(z), & z \in \mathfrak{J}_{i}, i=1,2, \ldots, m,\end{cases}
$$

where $\beta_{i}(z) \in M\left(\mathfrak{J}_{i}\right)(i=1,2, \ldots, m)$. Then the following three conditions are equivalent:

(a) $[\mu]_{T(\Delta)}=[\nu]_{T(\Delta)}$;

(b) $\left[\mu_{i}\right]_{T\left(\mathfrak{J}_{i}\right)}=\left[\beta_{i}\right]_{T\left(\mathfrak{J}_{i}\right)}$, where $\mu_{i}$ is the restriction of $\mu$ on $\mathfrak{J}_{i}(i=1,2, \ldots$, $m)$;

(c) $f^{\mu}(z)=f^{\nu}(z)$ for all $z$ on $\bigcup_{1}^{m} \partial \mathfrak{J}_{i}$.

Proof. $(\mathrm{a}) \Longrightarrow(\mathrm{c})$ : This is a direct corollary of Lemma 1. $(c) \Longrightarrow(b)$ : This follows from the definition of Teichmüller equivalence class. $(\mathrm{b}) \Longrightarrow(\mathrm{a})$ : Let $\left.f^{\mu}\right|_{\mathfrak{J}}: \mathfrak{J} \rightarrow f^{\mu}(\mathfrak{J})$ be the restriction of $f^{\mu}$ on $\mathfrak{J}$. Since $\left[\mu_{i}\right]_{T\left(\mathfrak{J}_{i}\right)}=\left[\beta_{i}\right]_{T\left(\mathfrak{J}_{i}\right)}$, by the definition of Teichmüller equivalence class and the Riemann mapping theorem, there exists a quasiconformal mapping $g_{i}$ from $\mathfrak{J}_{i}$ onto $f^{\mu}\left(\mathfrak{J}_{i}\right)$ such that the Beltrami coefficient $\mu_{g_{i}}$ of $g_{i}$ is $\beta_{i}(z)$ on $\mathfrak{J}_{i}(i=1,2, \ldots, m)$, which implies (a).

To obtain Theorem 2, we also need an infinitesimal version of Lemma 2.

Lemma 3. Let $\mathfrak{J}_{i}(i=1,2, \ldots, m)$, and let $\mathfrak{J}$ be given as above. Suppose that $\mu(z)$ is a Beltrami coefficient in $M(\Delta)$. Let $\nu(z)$ be another Beltrami coefficient in $M(\Delta)$ defined as follows:

$$
\nu(z)= \begin{cases}\mu(z), & z \in \Delta \backslash \mathfrak{J}, \\ \beta_{i}(z), & z \in \mathfrak{J}_{i}, i=1,2, \ldots, m,\end{cases}
$$

where $\beta_{i}(z) \in M\left(\mathfrak{J}_{i}\right)(i=1,2, \ldots, m)$. Then the following two conditions are equivalent:

(a) $[\mu]_{B(\Delta)}=[\nu]_{B(\Delta)}$;

(b) $\left[\mu_{i}\right]_{B\left(\mathfrak{J}_{i}\right)}=\left[\beta_{i}\right]_{B\left(\mathfrak{J}_{i}\right)}$, where $\mu_{i}$ is the restriction of $\mu$ on $\mathfrak{J}_{i}(i=1,2, \ldots$, $m)$.

Before proving Lemma 3, we introduce Lemma 5 of [1].

LEMMA 4 ([1, Lemma 5$])$. Let $\mathfrak{D} \subset \Delta$ be a subdomain such that $\overline{\mathfrak{D}} \subset \Delta$ and $\Delta-\overline{\mathfrak{D}}$ is connected and dense in $\Delta-\mathfrak{D}$. Then the restrictions to $\mathfrak{D}$ of quadratic differentials in $A(\Delta)$ are dense in $A(\mathfrak{D})$. 
Proof of Lemma 3. It is evident that (b) implies (a). We need to show only that (a) implies (b). If (a) holds, then for any $\varphi \in A(\Delta)$, we have

$$
\iint_{\Delta} \mu \varphi=\iint_{\Delta} \nu \varphi
$$

Because $\mu(z)=\nu(z)$ for $z \in \Delta \backslash \mathfrak{J}$, we have

$$
\iint_{\bigcup_{1}^{m} \mathfrak{J}_{i}} \mu \varphi=\iint_{\mathfrak{J}_{1}} \mu_{1} \varphi+\sum_{i=2}^{m} \iint_{\mathfrak{J}_{i}} \mu_{i} \varphi=\iint_{\mathfrak{J}_{1}} \beta_{1} \varphi+\sum_{i=2}^{m} \iint_{\mathfrak{J}_{i}} \beta_{i} \varphi .
$$

Applying Runge's theorem to $\overline{\mathfrak{J}_{i}}(i=1,2, \ldots, m)$, there exists a polynomial sequence $\left\{\psi_{n}\right\}$ such that

$$
\lim _{n \rightarrow \infty} \iint_{\mathfrak{J}_{i}}\left|\psi_{n}-\varphi\right|=0, \quad i=2, \ldots, m,
$$

and

$$
\lim _{n \rightarrow \infty} \iint_{\mathfrak{J}_{1}}\left|\psi_{n}\right|=0, \quad i=1
$$

Notice that

$$
\begin{aligned}
\iint_{\mathfrak{J}_{1}} \mu_{1}\left(\varphi-\psi_{n}\right)+\sum_{i=2}^{m} \iint_{\mathfrak{J}_{i}} \mu_{i}\left(\varphi-\psi_{n}\right)= & \iint_{\mathfrak{J}_{1}} \beta_{1}\left(\varphi-\psi_{n}\right) \\
& +\sum_{i=2}^{m} \iint_{\mathfrak{J}_{i}} \beta_{i}\left(\varphi-\psi_{n}\right) .
\end{aligned}
$$

Taking the limit on both sides of equality (3.3), we get

$$
\iint_{\mathfrak{J}_{1}} \mu_{1} \varphi=\iint_{\mathfrak{J}_{1}} \beta_{1} \varphi
$$

Furthermore, by Lemma 4 , for any $\phi \in A\left(\mathfrak{J}_{1}\right)$, we have

$$
\iint_{\mathfrak{J}_{1}} \mu_{1} \phi=\iint_{\mathfrak{J}_{1}} \beta_{1} \phi
$$

Namely, $\left[\mu_{1}\right]_{B\left(\mathfrak{J}_{1}\right)}=\left[\beta_{1}\right]_{B\left(\mathfrak{J}_{1}\right)}$. Similarly, $\left[\mu_{i}\right]_{B\left(\mathfrak{J}_{i}\right)}=\left[\beta_{i}\right]_{B\left(\mathfrak{J}_{i}\right)}(i=2, \ldots, m)$. Thus, the proof of Lemma 3 is complete. 


\section{$\S 4$. The construction theorem and its applications}

The following construction theorem is essentially the same as Reich's construction theorem in [6, page 343].

Construction theorem. Let $A$ be a compact subset of $\Delta$ consisting of $m(m \in \mathbb{N})$ connected components and such that $\Delta \backslash A$ is connected and each connected component of $A$ contains at least two points. There exists a function $\alpha \in E^{\infty}(\Delta)$ and a sequence $\varphi_{n} \in A(\Delta)(n=1,2, \ldots)$ satisfying these conditions:

$$
\begin{aligned}
|\alpha(z)| & = \begin{cases}0, & z \in A, \\
1, & \text { for a.a. } z \in \Delta \backslash A ;\end{cases} \\
\lim _{n \rightarrow \infty}\left\{\left\|\varphi_{n}\right\|-\lambda_{\alpha}\left[\varphi_{n}\right]\right\} & =0 ; \\
\lim _{n \rightarrow \infty}\left|\varphi_{n}(z)\right| & =\infty \text { a.e. in } \Delta \backslash A ;
\end{aligned}
$$

and as $n \rightarrow \infty$,

$$
\varphi_{n}(z) \rightarrow 0 \text { uniformly on } A \text {. }
$$

Proof. Reich's construction theorem gives the theorem when $m=1$. For simplicity and without loss of generality, we assume that $m=2$. Thus, since $A$ is compact and has two connected components, $\Delta \backslash A$ is triply connected. Let $X$ and $Y$ denote two connected components of $A$.

Let $\left\{J_{n}\right\},\left\{X_{n}\right\}$, and $\left\{Y_{n}\right\}$ be closed Jordan domains with the following properties:

$$
\begin{gathered}
J_{n} \subset \Delta, \quad J_{n} \subset \operatorname{Int}\left(J_{n+1}\right), \quad X_{n} \subset \Delta, \quad X_{n+1} \subset \operatorname{Int}\left(X_{n}\right), \quad Y_{n} \subset \Delta, \\
Y_{n+1} \subset \operatorname{Int}\left(Y_{n}\right), \quad J_{n} \cap X_{n}=\varnothing, \quad J_{n} \cap Y_{n}=\varnothing, \quad X_{n} \cap Y_{n}=\varnothing, \\
\left|\bigcup_{1}^{\infty} J_{n}\right|=|\Delta \backslash A|, \quad \bigcap_{1}^{\infty} X_{n}=X, \quad \bigcap_{1}^{\infty} Y_{n}=Y .
\end{gathered}
$$

The rest of the proof follows word for word from Reich [6, pages 343-346]. In addition, (4.4) is implied in his proof.

Combining the construction theorem and Lemma 1, we get the following. 
Lemma 5. Let $\mathfrak{J}_{i} \varsubsetneqq \Delta(i=1,2, \ldots, m)$ be $m$ Jordan domains such that $\overline{\mathfrak{J}_{i}}(i=1,2, \ldots, m)$ are mutually disjoint and $\Delta \backslash \bigcup_{1}^{m} \overline{\mathfrak{J}_{i}}$ is connected. Let $A=\bigcup_{1}^{m} \overline{\mathfrak{J}_{i}}$. Suppose that $\alpha(z)$ and the sequence $\varphi_{n} \in A(\Delta)$ are constructed by the construction theorem, and let $\mu(z)=k \alpha(z)$, where $k<1$ is a positive constant. Set

$$
\nu(z)= \begin{cases}\mu(z), & z \in \Delta \backslash A \\ \beta_{i}(z), & z \in \mathfrak{J}_{i}, i=1,2, \ldots, m\end{cases}
$$

where $\beta_{i}(z)$ is in $M\left(\mathfrak{J}_{i}\right)$ with $\left\|\beta_{i}\right\|_{\infty} \leq k(i=1,2, \ldots, m)$. Then

(1) $\nu(z)$ is extremal in $[\nu]_{T(\Delta)}$, and for any $\chi(z)$ in $[\nu]_{T(\Delta)}^{*}, \chi(z)=\nu(z)$ for almost all $z$ in $\Delta \backslash A$;

(2) $\nu(z)$ is extremal in $[\nu]_{B(\Delta)}$, and for any $\chi(z)$ in $[\nu]_{B(\Delta)}^{*}, \chi(z)=\nu(z)$ for almost all z in $\Delta \backslash A$.

Proof. Obviously, $\|\mu\|_{\infty}=\|\nu\|_{\infty}=k$. Set $E=\Delta \backslash A$. Notice that the sequence $\varphi_{n}(z)$ satisfies conditions (4.2)-(4.4). We have

$$
\lim _{n \rightarrow \infty} \iint_{A}\left|\varphi_{n}(z)\right| d x d y=0
$$

and hence

$$
\lim _{n \rightarrow \infty} \iint_{A} \beta(z) \varphi_{n}(z) d x d y=0 .
$$

Furthermore, by

$$
k \iint_{E}\left|\varphi_{n}(z)\right| d x d y-\operatorname{Re} \iint_{E} \mu(z) \varphi_{n}(z) d x d y \leq\left\|\varphi_{n}\right\|-\lambda_{\alpha}\left[\varphi_{n}\right],
$$

we achieve

$$
\begin{aligned}
\lim _{n \rightarrow \infty}\left(k \iint_{\Delta}\left|\varphi_{n}(z)\right| d x d y-\operatorname{Re} \iint_{\Delta} \nu(z) \varphi_{n}(z) d x d y\right) \\
=\lim _{n \rightarrow \infty}\left(k \iint_{E}\left|\varphi_{n}(z)\right| d x d y-\operatorname{Re} \iint_{E} \mu(z) \varphi_{n}(z) d x d y\right) \\
\quad+\lim _{n \rightarrow \infty}\left(k \iint_{A}\left|\varphi_{n}(z)\right| d x d y-\operatorname{Re} \iint_{A} \beta(z) \varphi_{n}(z) d x d y\right)=0
\end{aligned}
$$

In short,

$$
\lim _{n \rightarrow \infty}\left(k\left\|\varphi_{n}\right\|-\lambda_{\nu}\left[\varphi_{n}\right]\right)=0
$$


Thus, by (4.3) and Fatou's lemma,

$$
k-\operatorname{Re} \iint_{\Delta} \nu(z) \frac{\varphi_{n}(z)}{\left\|\varphi_{n}\right\|} \longrightarrow 0, \quad n \rightarrow \infty,
$$

which shows that $\nu(z)$ is extremal in $[\nu]_{T(\Delta)}$ and hence is extremal in $[\nu]_{B(\Delta)}$.

(1) Assume that $\chi(z)$ is extremal in $[\nu]_{T(\Delta)}$, that is, that $\chi(z) \in[\nu]_{T(\Delta)}^{*}$. Let $\widetilde{\nu}(w), \widetilde{\chi}(w)$ denote the Beltrami coefficients of $\left(f^{\nu}\right)^{-1}$ and $\left(f^{\chi}\right)^{-1}$, respectively. We claim that $\widetilde{\nu}\left(f^{\nu}(z)\right)=\widetilde{\chi}\left(f^{\nu}(z)\right)$ for almost every $z \in \Delta \backslash A$. Suppose the contrary case; then there would exist $\epsilon>0$ and a compact subset $S$ of $\Delta \backslash A$ with positive Lebesgue measure such that $\mid\left(\widetilde{\nu}\left(f^{\nu}\right)-\widetilde{\chi}\left(f^{\nu}\right)\right) /(1-$ $\left.\overline{\widetilde{\nu}\left(f^{\nu}\right)} \widetilde{\chi}\left(f^{\nu}\right)\right) \mid \geq \epsilon>0$ on $S$. Then, by the delta inequality (2.3), there exists a positive constant $C$ depending only on $k$ such that

$$
\iint_{\Delta}\left|\frac{\widetilde{\nu}\left(f^{\nu}\right)-\widetilde{\chi}\left(f^{\nu}\right)}{1-\overline{\widetilde{\nu}\left(f^{\nu}\right)} \widetilde{\chi}\left(f^{\nu}\right)}\right|^{2}\left|\varphi_{n}\right| \leq C\left(k\left\|\varphi_{n}\right\|-\operatorname{Re} \iint_{\Delta} \nu \varphi_{n}\right) .
$$

Therefore,

$$
\frac{\epsilon^{2}}{4} \iint_{S}\left|\varphi_{n}\right| \leq C\left(k\left\|\varphi_{n}\right\|-\operatorname{Re} \iint_{\Delta} \nu \varphi_{n}\right)=C\left(k\left\|\varphi_{n}\right\|-\lambda_{\nu}\left[\varphi_{n}\right]\right) .
$$

By (4.3) and Fatou's lemma, the left-hand side of the above inequality has a positive lower bound, while by (4.5), the right-hand side tends to zero as $n \rightarrow \infty$. This contradiction induces our claim.

Applying Lemma 1 to $\widetilde{\mathfrak{J}}=f^{\nu}\left(\bigcup_{1}^{m} \mathfrak{J}_{i}\right)$ on the target unit disk, we find that $\left(f^{\nu}\right)^{-1}(w)=\left(f^{\chi}\right)^{-1}(w)$ for all $w$ in $f^{\nu}(\bar{E})$ and that $\left(f^{\nu}\right)^{-1}(\widetilde{\mathfrak{J}})=\left(f^{\chi}\right)^{-1}(\widetilde{\mathfrak{J}})$. In other words, $f^{\nu}(z)=f^{\chi}(z)$ for all $z$ in $\bar{E}$. Therefore, $\nu(z)=\chi(z)$ for almost every $z$ in $\bar{E}$.

(2) Applying the infinitesimal delta inequality, one easily shows that $\nu(z)$ is extremal in $[\nu]_{B(\Delta)}$ and that, for any $\chi(z)$ in $[\nu]_{B(\Delta)}^{*}$, we have $\chi(z)=\nu(z)$ for almost all $z$ in $\Delta \backslash A$. We skip the details here.

\section{$\S 5$. Proof of the main results}

To prove our main results, it suffices to construct $\mu \in M(\Delta)$ such that $[\mu]_{T(\Delta)}^{*}$ or $[\nu]_{B(\Delta)}^{*}$ contains more than one extremal Beltrami coefficient and contains no extremal Beltrami coefficients of constant modulus.

Proof of Theorem 1. Suppose that $m \geq 2$. Let $\mathfrak{J}_{i}(i=1,2, \ldots, m)$ be given as in Lemma 5. Choose $A=\bigcup_{1}^{m} \overline{\mathfrak{J}_{i}}$. Let $\alpha(z)$ and the sequence $\varphi_{n} \in A(\Delta)$ 
be constructed by the construction theorem, and let $\mu(z)=k \alpha(z)$, where $k<1$ is a positive constant.

By the counterexample theorem in [1, Theorem 10], there exists a Beltrami coefficient $\beta_{1}(z)$ in $M\left(\mathfrak{J}_{1}\right)$ with $\left\|\beta_{1}\right\|_{\infty}=k$ such that $\beta_{1}$ is uniquely extremal in $\left[\beta_{1}\right]_{T\left(\mathfrak{J}_{1}\right)}$ and $\left|\beta_{1}\right|$ is not almost everywhere constant on $\mathfrak{J}_{1}$. Now, set

$$
\nu(z)= \begin{cases}\mu(z), & z \in \Delta-\bigcup_{1}^{m} \mathfrak{J}_{i}, \\ \beta_{1}(z), & z \in \mathfrak{J}_{1}, \\ \beta_{i}(z), & z \in \mathfrak{J}_{i}, 1<i \leq m\end{cases}
$$

where $\beta_{2}(z) \in M\left(\mathfrak{J}_{2}\right)$ is chosen with $\left\|\beta_{2}\right\|_{\infty}<k$, and $\beta_{i}(z) \in M\left(\mathfrak{J}_{i}\right)$ is chosen with $\left\|\beta_{i}\right\|_{\infty} \leq k(i \neq 1,2)$. Then $\nu(z)$ is extremal in $[\nu]_{T(\Delta)}$ in virtue of Lemma 5 but is not uniquely extremal for $\left\|\beta_{2}\right\|_{\infty}<k$ on $\mathfrak{J}_{2}$.

We continue to show that $[\nu]_{T(\Delta)}$ contains no extremal Beltrami coefficients of constant modulus. Suppose that $\gamma(z) \in[\nu]_{T(\Delta)}$ is extremal. Then $|\gamma(z)| \leq k$ for almost all $z$ in $\Delta$.

On the other hand, combining Lemma 5 and Lemma 2, we have

$$
\left[\beta_{1}\right]_{T\left(\mathfrak{J}_{1}\right)}=\left[\left.\gamma\right|_{\mathfrak{J}_{1}}\right]_{T\left(\mathfrak{J}_{1}\right)},
$$

where $\left.\gamma\right|_{\mathfrak{J}_{1}}$ is the restriction of $\gamma$ on $\mathfrak{J}_{1}$.

Notice that $\beta_{1}$ is uniquely extremal in $\left[\beta_{1}\right]_{T\left(\mathfrak{J}_{1}\right)}$ with $\left\|\beta_{1}\right\|_{\infty}=k$ and that $\left|\beta_{1}\right|$ is not almost everywhere constant on $\mathfrak{J}_{1}$. We find that $\gamma(z)=\beta_{1}(z)$ for almost all $z \in \mathfrak{J}_{1}$. Thus, we prove that for any $\gamma(z)$ extremal in $[\nu]_{T(\Delta)},|\gamma|$ is not almost everywhere constant on $\mathfrak{J}_{1}$.

This completes the proof of Theorem 1 .

Proof of Theorem 2. For simplicity, we use the same denotations as in the proof of Theorem 1. We only need to show that $[\nu]_{B(\Delta)}$ satisfies the requirement of Theorem 2 , where $\nu(z)$ is constructed by $(5.1)$.

It follows that $\nu$ is extremal in $[\nu]_{B(\Delta)}^{*}$ from Lemma 5 and is not uniquely extremal by the equivalence theorem in [1]. Suppose that $\gamma(z) \in[\nu]_{B(\Delta)}$ is extremal. Then $|\gamma(z)| \leq k$ for almost all $z$ in $\Delta$.

Because $\beta_{1}$ is uniquely extremal in $\left[\beta_{1}\right]_{T\left(\mathfrak{J}_{1}\right)}$ with $\left\|\beta_{1}\right\|_{\infty}=k, \beta_{1}$ is uniquely extremal in $\left[\beta_{1}\right]_{B\left(\mathfrak{J}_{1}\right)}$ again by the equivalence theorem.

On the other hand, combining Lemma 5 and Lemma 3, we have

$$
\left[\beta_{1}\right]_{B\left(\mathfrak{J}_{1}\right)}=\left[\left.\gamma\right|_{\mathfrak{J}_{1}}\right]_{B\left(\mathfrak{J}_{1}\right)}
$$


where $\left.\gamma\right|_{\mathfrak{J}_{1}}$ is the restriction of $\gamma$ on $\mathfrak{J}_{1}$. We again find that $\gamma(z)=\beta_{1}(z)$ for almost all $z \in \mathfrak{J}_{1}$. Notice that $\left|\beta_{1}\right|$ is not almost everywhere constant on $\mathfrak{J}_{1}$. Thus, we prove, for any $\gamma(z)$ extremal in $[\nu]_{B(\Delta)},|\gamma|$ is not almost everywhere constant on $\mathfrak{J}_{1}$. Namely, $[\nu]_{B(\Delta)}$ contains no extremal Beltrami coefficients of constant modulus. The proof of Theorem 2 is complete.

We do not know whether there is some essential relation between $[\mu]_{T(\Delta)}$ and $[\mu]_{B(\Delta)}$ if $[\mu]_{T(\Delta)}$ or $[\mu]_{B(\Delta)}$ contains an extremal Beltrami coefficient of constant modulus. The following problem might be interesting.

Problem 1. Suppose that $\mu$ is an extremal Beltrami coefficient. If $[\mu]_{T(\Delta)}$ contains an extremal Beltrami coefficient of constant modulus, does it imply that $[\mu]_{B(\Delta)}$ does also? What about the converse?

REMARK 2. If $\mu$ itself is of constant modulus, the answer is a fortiori. Recently, Fan and Chen [3] gave a negative answer to the above problem in virtue of the method used in this paper if $\mu$ need not be extremal.

\section{$\S 6$. On the measure of extremal sets}

Suppose that $\mu$ is an extremal Beltrami coefficient. For any $\eta$ extremal in $[\mu]_{T(\Delta)}\left(\right.$ or $\left.[\mu]_{B(\Delta)}\right)$, let $X[\eta]=\left\{z \in \Delta:|\eta(z)|=\|\mu\|_{\infty}\right\}$. We call $X[\eta]$ the extremal set of $\eta$.

Suppose that $\nu$ is constructed as (5.1) in the proof of Theorem 1. Let $l=\operatorname{mes}\left(\left.X\right|_{\mathfrak{J}_{1}}\left[\beta_{1}\right]\right)$ be the Lebesgue measure of the extremal set $\left.X\right|_{\mathfrak{J}_{1}}\left[\beta_{1}\right]=$ $\left\{z \in \mathfrak{J}_{1}:\left|\beta_{1}(z)\right|=\left\|\beta_{1}\right\|_{\infty}=k\right\}$. Thus, in virtue of the proof of Theorem 1 (or Theorem 2), for any extremal Beltrami coefficient $\eta$ in $[\nu]_{T(\Delta)}\left(\right.$ or $\left.[\nu]_{B(\Delta)}\right)$, $X[\eta]$ satisfies

$$
l+\pi-\sum_{i=1}^{m} \operatorname{mes}\left(\mathfrak{J}_{i}\right) \leq \operatorname{mes}(X[\eta]) \leq l+\pi-\operatorname{mes}\left(\mathfrak{J}_{1}\right) .
$$

Therefore, we have proved the following.

Corollary 3. Suppose that $s, t \in[0, \pi]$ are two arbitrarily given constants with $s<t$. Then there exists $[\mu]_{T(\Delta)} \in T(\Delta) \quad\left(\right.$ or $[\mu]_{B(\Delta)} \in B(\Delta)$ ) such that $[\mu]_{T(\Delta)}^{*}$ (or $\left.[\mu]_{B(\Delta)}^{*}\right)$ contains infinitely many elements, and such that for any $\eta \in[\mu]_{T(\Delta)}^{*}\left(\right.$ or $\left.[\mu]_{B(\Delta)}^{*}\right), s \leq \operatorname{mes}(X[\eta]) \leq t$.

Corollary 3 actually solves two problems about the measure of extremal sets posed in [12]. Naturally, it is interesting to consider the special case 
that the extremal sets of all elements in $[\mu]_{T(\Delta)}^{*}\left([\mu]_{B(\Delta)}^{*}\right)$ have the same measure. Precisely, we pose the following.

Problem 2. Suppose that for any extremal Beltrami coefficient $\eta$ in $[\mu]_{T(\Delta)}\left(\right.$ or $\left.[\mu]_{B(\Delta)}\right)$, $\operatorname{mes}(X[\eta])=s$, where $s \in[0, \pi]$ is a constant. Does it imply that $[\mu]_{T(\Delta)}^{*}\left(\right.$ or $\left.[\mu]_{B(\Delta)}^{*}\right)$ contains only one element (i.e., a uniquely extremal one)?

If $[\mu]_{T(\Delta)}\left([\mu]_{B(\Delta)}\right)$ contains infinitely many extremal Beltrami coefficients, then there exists at least an extremal Beltrami coefficient in $[\mu]_{T(\Delta)}$ $\left([\mu]_{B(\Delta)}\right)$ with nonconstant modulus. The result is actually implied by Reich's proof of his theorem [7, page 34] (also see [12, Theorem 1]). A similar discussion related to the result was earlier given by Mateljević and Marković [4]. It was proved for the case of more general hyperbolic Riemann surfaces in [11]. Thus, we have an affirmative answer to Problem 2 when $s=\pi$, the remaining cases of which are open. We further believe that, in the setting of being nonuniquely extremal, $[\mu]_{T(\Delta)}\left([\mu]_{B(\Delta)}\right)$ contains infinitely many extremal coefficients with nonconstant modulus; moreover, if $[\mu]_{T(\Delta)}\left([\mu]_{B(\Delta)}\right)$ admits an extremal with constant modulus, then it admits infinitely many. However, we have no proof of this up to the present.

Acknowledgments. The author would like to express gratitude to the referee for his valuable comments. Thanks are also due Professor Edgar Reich for his careful reading of this paper and for giving useful suggestions.

\section{REFERENCES}

[1] V. Božin, N. Lakic, V. Marković, and M. Mateljević, Unique extremality, J. Anal. Math. 75 (1998), 299-338.

[2] C. J. Earle and N. Lakic, Variability sets on Riemann surfaces and forgetful maps between Teichmüller spaces, Ann. Acad. Sci. Fenn. Math. 27 (2002), 307-324.

[3] J. Fan and J. Chen, On the equivalence of extremal Teichmüller mapping, Sci. China Ser. A. 52 (2009), 77-86.

[4] V. Mateljević and V. Marković, The unique extremal QC mapping and uniqueness of Hanh-Banach extensions, Mat. Vesnik 48 (1996), 107-112.

[5] E. Reich, A criterion for unique extremality of Teichmüller mappings, Indiana Univ. Math. J. 30 (1981), 441-447.

[6] E. Reich, The unique extremality counterexample, J. Anal. Math. 75 (1998), 339-347.

[7] E. Reich, Non-uniquely extremal quasiconformal mappings, Libertas Math. 20 (2000), 33-38.

[8] E. Reich and K. Strebel, "Extremal quasiconformal mappings with given boundary values" in Contributions to Analysis: A Collection of Papers Dedicated to Lipman Bers, Academic Press, New York, 1974, 375-391. 
[9] K. Strebel, Point shift differentials and extremal quasiconformal mappings, Ann. Acad. Sci. Fenn. Math. 23 (1998), 475-494.

[10] G. W. Yao, Is there always an extremal Teichmüller mapping? J. Anal. Math. 94 (2004), 363-375.

[11] G. W. Yao and Y. Qi, On the modulus of extremal Beltrami coefficients, J. Math. Kyoto Univ. 46 (2006), 235-247.

[12] Z. Zhou, J. Chen, and Z. Yang, On the extremal sets of extremal quasiconformal mappings, Sci. China Ser. A 46 (2003), 552-561.

Guowu Yao

Department of Mathematical Sciences

Tsinghua University

Beijing, 100084

People's Republic of China

gwyao@math.tsinghua.edu.cn 